\title{
Myeloid-Derived Suppressor Cells in Pregnancy and the Neonatal Period
}

\author{
Natascha Köstlin-Gille and Christian Gille * \\ Department of Neonatology, University Children's Hospital Tuebingen, Tübingen, Germany
}

During pregnancy, the immune systems of mother and offspring are challenged by their close adjacency to balance tolerance and rejection. After birth the neonate has to continue this balance towards its new environment by tolerating commensals while rejecting pathogens and towards its developing tissues to avoid inflammatory damage while overcoming immunosuppression. Our group was the first to link immunosuppressive features of myeloid derived suppressor cells (MDSC) to materno-fetal tolerance, neonatal susceptibility to infection and inflammation control. Here we summarize recent advances in this dynamic field.

\section{OPEN ACCESS}

Edited by:

David J. Dowling,

Boston Children's Hospital,

United States

Reviewed by:

Alain Le Moine,

Université Libre de Bruxelles, Belgium

Huanfa Yi,

Jilin University, China

*Correspondence:

Christian Gille

christian.gille@med.uni-tuebingen.de

Specialty section:

This article was submitted to

Nutritional Immunology,

a section of the journal

Frontiers in Immunology

Received: 17 July 2020

Accepted: 21 September 2020

Published: 09 October 2020

Citation:

Köstlin-Gille N and Gille C (2020) Myeloid-Derived Suppressor Cells in Pregnancy and the Neonatal Period.

Front. Immunol. 11:584712.

doi: 10.3389/fimmu.2020.584712
Keywords: myeloid-derived suppressor cells (MDSC), pregnancy, materno-fetal tolerance, neonate, tolerance, microbiome

\section{INTRODUCTION}

Discrimination between self and non-self is one of the fundamental features of the mammalian immune system. Especially during pregnancy, the immune systems of both, the mother and the fetus perfectly balance between protection against pathogens and tolerance towards a semiallogeneic organism. Dysfunction of the immune adaptation during pregnancy can lead to severe complications like pregnancy loss, preeclampsia, preterm birth or fetal growth restriction. Initially the field of materno-fetal tolerance mainly focused on T cell immunology (1) [reviewed in (2)], however it has become more and more clear that the immune mechanisms leading to successful pregnancy are much more complex and that our understanding of how this exceptional situation is facilitated exhibits significant gaps.

After birth, the neonatal organism is challenged to adapt immunological functions, when body surfaces become colonized with microbes, directly exposing the neonatal immune system to potential pathogens, also requiring a perfect balance between defense against pathogens and tolerance towards commensals.

Myeloid-derived suppressor cells (MDSC) are myeloid cells with the ability to suppress various types of immune responses. While other myeloid cells such as monocytes, macrophages, dendritic cells (DCs), polymorphnuclear (PMN) neutrophils, eosinophils, and basophils classically get activated by strong signals through pathogen-associated molecular patterns (PAMPs) or dangerassociated molecular patterns (DAMPs) resulting in a pro-inflammatory response, MDSC rather expand under conditions with chronic infection or inflammation and act anti-inflammatory (3). MDSC mainly consist of two cell types named granulocytic MDSC (GR-MDSC) with phenotypic similarity to neutrophils and monocytic MDSC (MO-MDSC) with phenotypic similarity to 
monocytes, to date making it impossible to clearly identify MDSC only by phenotypic characteristics. In mice, GR-MDSC

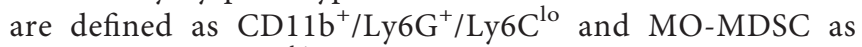

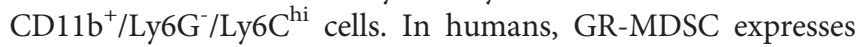
the granulocytic markers CD11b, CD15 and/or CD66b, but are negative for the monocytic surface antigen CD14. They can only be distinguished from granulocytes by their sedimentation in the low-density fraction (peripheral blood mononuclear cells, PBMC) after density gradient centrifugation. Recent data suggest, that expression of the lectin-type oxidized low density lipoprotein (LDL) receptor 1 (LOX-1) might be a possible marker to distinguish between granulocytes and GR-MDSC. Human MO-MDSC are defined as CD14 $/ 4_{\text {HAA-DR }}{ }^{\mathrm{lo} /-}$; their discrimination from monocytes is based on their generally lower expression of HLA-DR (4-6). However to date, GR-MDSC and MO-MDSC have to be identified by their suppressive capacity, primarily towards $\mathrm{T}$-cell proliferation.

MDSC use a large number of mechanisms to suppress immune responses. Among these, the best known are a depletion of the essential amino acid arginine by expression of the enzymes Arginase I (ArgI) and inducible nitric oxide synthase (iNOS), the sequestration of cysteine by indoleamine 2,3-dioxygenase (IDO), the production of anti-inflammatory cytokines like TGF $\beta$ and IL-10, the synthesis of prostaglandins by expression of cyclooxygenase- 2 (COX-2) and the production of reactive oxygen species (ROS) [reviewed in (7)]. The main targets of MDSC-mediated immune suppression are T-cells, however also a suppression of NK-cell functions (8) and DC functions (9), as well as a modulation of monocyte/macrophage functions (10) have been reported. Furthermore, MDSC can induce regulatory T-cells (11), which in turn exert their own type of immune suppression.

Accumulation and activation of MDSC are driven by various factors. Condamine et al. proposed a two-signal model in which the first signal leads to an expansion of myeloid cells and inhibition of their differentiation and the second signal converts these immature cells to $\operatorname{MDSC}(3,12)$. Factors involved in this process are growth factors and cytokines leading to activation of transcription factors such as signal transducer and activator of transcript 1, 3 and 6 (STAT1, 3, and 6), CCAAT/enhancer binding protein $\beta(\mathrm{C} / \mathrm{EBP} \beta)$ or NOD-, LRR- and pyrin-domain containing protein 3 (NLRP3) [reviewed in (12)].

Primarily, MDSC accumulation has been described under tumor conditions, leading to inhibition of the immune response against tumor cells and to spreading of the disease (13). In the following years, MDSC accumulation has been described under various other conditions such as sepsis/infection, trauma, autoimmune diseases, obesity, ageing and transplantation where they seem to play either a detrimental or a beneficial role [reviewed in (14)]. Overall, it appears that under conditions, where immune tolerance is needed to survive, MDSC accumulation may be advantageous for the host. In this review, we aim to summarize data on MDSC during perinatal time, i.e. during pregnancy and the neonatal period, as a phase of life, where under physiological conditions tolerance is needed most.

\section{MDSC DURING PREGNANCY}

\section{MDSC During Normal Pregnancy}

The first description of a MDSC-accumulation during pregnancy came from Mauti et al. who showed that increased permissiveness for tumor metastasis during gestation in mice was accompanied by an expansion of MDSC with inhibitory effects on NK-cell activity and that depletion of MDSC in pregnant mice reduced metastasis (8). Later, our group showed, that also during physiologic conditions in healthy human pregnancies an accumulation of GR-MDSC but not MO-MDSC occurred with up to tenfold higher numbers of GR-MDSC in the peripheral blood of pregnant women compared to blood of healthy non-pregnant controls (15). Levels of GR-MDSC were highest during early gestation (15, 16) and dropped within a few days postpartum to levels of nonpregnant women (15). In human placenta GR-MDSC were shown to be enriched in comparison to maternal and fetal blood (17) and mainly located in decidua and intervillous space $(17,18)$. Genetic analyses revealed that they descend from maternal origin (17). Pan et al. and Zhang et al., showed that also MO-MDSC increased in the peripheral blood of pregnant women $(19,20)$, however percentages of MO-MDSC were much lower than that of GR-MDSC.

In accordance with the data from humans, it has been shown that GR-MDSC also expand during murine pregnancy both in the periphery $(21,22)$ and in the uterus (22-24), especially during early gestation $(21,24)$. This phenomenon was observed in syngeneic $(8,22,23)$ as well as in allogeneic $(21$, $24,25)$ murine pregnancies. Mouse strains used were BALB/c (21), C57BL/6J (8, 22, 25), CBA/J (24, 25), and FBVn (23).

\section{MDSC During Pathological Pregnancies}

It was shown that women with miscarriage had decreased levels of GR-MDSC both in blood (16) and in placenta $(16,17,26)$, while numbers of MO-MDSC did not differ (26). Studies in mice revealed that MDSC accumulation during pregnancy was also decreased in abortion prone animals $(24,25,27)$ and adoptive transfer prevented fetal rejection in the murine abortion model (25). In addition, depletion of MDSC caused gestation failure $(21,23-25)$. Interestingly, Ostrand-Rosenberg et al. showed that especially MDSC-depletion at day 4.5 (E4.5) of murine pregnancy, which is the time of implantation, completely prevented successful pregnancy, while MDSC-depletion after E8.5 did not affect pregnancy rates (21). This is in line with two human studies showing that high GR-MDSC levels predict a better outcome after in-vitro fertilization $(28,29)$. One study investigated MDSC in preeclampsia and showed that GR-MDSC, but not MO-MDSC levels are decreased in peripheral blood and cord blood of preeclampsia patients in comparison to healthy pregnancies (30), however functional studies are lacking.

\section{MDSC Functions and Suppressive Mechanisms During Pregnancy}

Pregnancy induced MDSC from different compartments (peripheral blood, uterus, decidua) exert different effector 
mechanisms to modulate immune effector cells during pregnancy. A summary is depicted in Table $\mathbf{1}$ and Figure $\mathbf{1}$.

\section{Inhibition of T-Cell Function}

Suppression of T-cell function is a main feature of MDSC. Changes in $\mathrm{T}$-cell function are one of the best examined mechanisms mediating maternal-fetal tolerance (33). In humans, it could have been shown that GR-MDSC during pregnancy inhibit $\mathrm{T}$-cell proliferation $(15,17,18)$, express ArgI, iNOS and IDO and produce ROS (15, 18, 30). ArgI expression led to downregulation of $\mathrm{CD} 3 \zeta$ chain on $\mathrm{T}$ cells

TABLE 1 | Origin, effects and mechanisms of MDSC in pregnancy.

\begin{tabular}{|c|c|c|c|}
\hline MDSC origin & Effect & $\begin{array}{l}\text { Main } \\
\text { mechanism } \\
\text { of MDSC }\end{array}$ & Ref. \\
\hline \multirow[t]{3}{*}{$\begin{array}{l}\text { GR-MDSC from } \\
\text { peripheral blood }\end{array}$} & Inhibition of T-cell proliferation & - & $\begin{array}{l}(15, \\
17,18, \\
31)\end{array}$ \\
\hline & Downregulation of CD3 $\zeta$ chain & Argl & (31) \\
\hline & Inhibition of T-cell proliferation & ROS & (25) \\
\hline \multirow{3}{*}{$\begin{array}{l}\text { GR-MDSC from } \\
\text { placenta } \\
\text { (human) }\end{array}$} & Inhibition of Th1 response & Cell contact & $(17)$ \\
\hline & Induction of Th2 response & Cell contact & $(17)$ \\
\hline & Inhibition of T-cell proliferation & ROS & $(25)$ \\
\hline \multirow[t]{5}{*}{ MDSC (murine) } & Inhibition of T-cell proliferation & ROS & $(25)$ \\
\hline & Decrease of uterine T-cells & - & $(21,23)$ \\
\hline & $\begin{array}{l}\text { Decrease of T-cell activation, L- } \\
\text { selectin downregulation }\end{array}$ & - & $(21)$ \\
\hline & Induction of Tregs & $\begin{array}{l}\text { TGF-beta } \\
\text { Beta-catenin }\end{array}$ & $(24)$ \\
\hline & $\begin{array}{l}\text { Reduce NK-cell cytotoxicity, inhibition } \\
\text { of perforin, downregulation of } \\
\text { NKG2D }\end{array}$ & - & $(8,32)$ \\
\hline
\end{tabular}

Source of MDSC, effect on immune effector cell and the main MDSC mechanisms are listed. Argl, arginase l; ROS, reactive oxygen species; iNOS, inducible NO-synthetase. and to decreased T-cell proliferation (31), while inhibition of ArgI restored T-cell proliferation in-vitro (17). Furthermore, it was shown that GR-MDSC isolated from placenta exhibited marked increased ROS-production in comparison to GRMDSC from the periphery (17). In pregnant mice, the main mechanism of T-cell suppression by GR-MDSC was ROSproduction (25). From tumor-bearing mice, it is known that induction of antigen-specific T-cell tolerance is mediated by MDSC via ROS (34), indicating that increased ROS-production could be also a mechanism for suppression of specific T-cellimmunity against fetal antigens.

In mice, Zhao et al. showed that depletion of MDSC during pregnancy led to an increase in uterine T-cells (23). Later, OstrandRosenberg et al. showed, that the detrimental effect of MDSCdepletion during pregnancy was mediated by an increase in T-cell activation and an upregulation of L-Selectin supposing that MDSC prevent homing of alloreactive T-cells to the uterus (21).

\section{Modulation of T-Helper Cell Function}

Besides a general suppression of T-cell responses, MDSC during pregnancy also modulate polarization of Th-cells. Different studies described a predominance of Th2 responses and a suppression of Th1-responses [reviewed in (35)] as well as an accumulation of Tregs during normal pregnancy (36). Our group showed in-vitro that placental GR-MDSC induce Th2-responses and inhibit Th1responses in a cell-contact dependent manner (17). Furthermore, Kang et al. showed that GR-MDSC from pregnant mice induced Tregs via production of TGF- $\beta$ and the transcription regulator $\beta$ catenin (24). In contrast to that, Ren et al. described an increase in Tregs after MDSC-depletion during pregnancy.

\section{Inhibition of NK Cell Function}

NK cells are the predominant cell type in deciduae of early pregnancies [reviewed in $(37,38)]$ and MDSC have been shown to suppress NK-cell functions under tumor-conditions $(39,40)$. During pregnancy, MDSC may contribute to reduced NK-cell

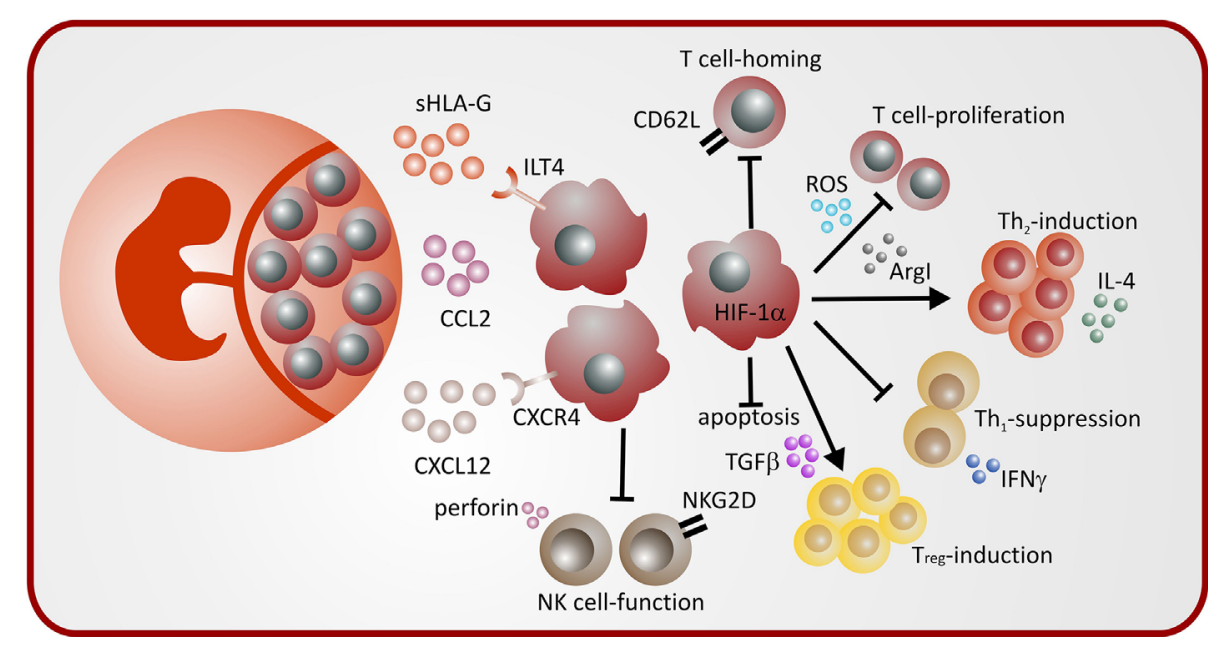

FIGURE 1 | Mechanisms of MDSC induction and MDSC-mediated immune modulation during pregnancy. 
cytotoxicity systemically (8), as well as locally in the uterus (32). Mechanisms for inhibition of NK cell function by MDSC during pregnancy are an inhibition of perforin-cytotoxicity and a downregulation of the surface receptor NKG2D on NK-cells (32).

\section{Effects on Myeloid Cells}

Decidual DCs have been shown to be in a tolerogenic state with reduced co-stimulatory capacity and altered cytokine expression [reviewed in (41)]. Hu et al. showed that in hepatocellular carcinoma MDSC impair DC function (42). To our knowledge, until now no data exists concerning the influence of MDSC on myeloid cell function during pregnancy. Zhao et al. described an increase in infiltrating DCs in the uteri of pregnant animals after MDSC-depletion, while total myeloid cell numbers remained unchanged (23), however functional data is missing.

\section{MDSC Expansion and Activation During Pregnancy \\ Soluble Factors Regulating MDSC Expansion and Activation During Pregnancy}

Different studies investigated potential mechanisms regulating MDSC accumulation and activation during pregnancy. Zhang et al. showed in vitro and in human cells an expansion of and an upregulation of Arg1 in MO-MDSC by the human trophoblast cell line HTR8 via CCL2 (20). Pan et al. showed an induction of MO-MDSC by $17 \beta$-Estradiol also in human cells (19). In murine cells, progesterone but not estrogen induced MDSC in-vitro. Our group showed that co-culture with the human trophoblast cell line JEG-3 induced MDSC. This was partially mediated through the receptor CXCR4 on MDSC, indicating that CXCL12, the ligand of CXCR4, plays a role in MDSC-expansion (17). Furthermore, we showed that soluble human leucocyte antigen G (sHLA-G) induced MDSC from PBMC through the receptor immunoglobulin like transcript (ILT) 4, increased suppressive activity of GR-MDSC and induced IDO expression (43).

In-vivo, Ostrand-Rosenberg et al. demonstrated that the IDOinhibitor 1-methyltryptophan (1-MT) decreased the level of MDSC in pregnant mice, while application of granulocytic colony-stimulating factor (G-CSF) restored MDSC-levels (21). Studies describing soluble factors mediating MDSC induction or activation in-vivo that may be targets for therapeutic interventions are still lacking.

\section{Transcriptional Regulation of MDSC Expansion and Activation During Pregnancy}

Three studies described a role of STAT3 for regulation of MDSC function during pregnancy. STAT3 has been shown to be involved in MDSC expansion via estradiol and via progesterone $(19,25)$ as well as in HLA-G-mediated MDSC-activation (43). Furthermore, our group showed that lack of the transcription factor hypoxiainducible factor $1 \alpha$ (HIF-1 $\alpha)$ led to decreased accumulation of MDSC during pregnancy and impaired suppressive activity (22). Interestingly, mice with homozygous deletion of heme oxygenase 1 (HO-1), one of the target genes of HIF-1 $\alpha$ (44) are infertile and heterozygous deletion results in preeclampsia like pregnancies with primarily absorption of HO-1-deficient fetuses $(45,46)$.
Furthermore, it has been shown that HO-1 is relevant for MDSC function during transplantation (47). The role of HO-1 for MDSC function during pregnancy remains to be elucidated. A summary of mechanisms leading to MDSC accumulation and activation during pregnancy is depicted in Figure $\mathbf{1 .}$

\section{MDSC IN THE FETUS AND NEONATE}

\section{MDSC in Cord Blood}

Concordant to the maternal side, MDSC have also been found in cord blood, suggesting that they not only modulate maternal but also fetal immune system to ensure feto-maternal tolerance (48). In two cohorts comprising cord blood samples from 83 healthy, full-term neonates after caesarian section, MDSC accounted for about 5\% $(0,3 \%-60 \%)$ of mononuclear cells $(48,49)$ - levels formerly only described in pathological processes. Accumulated cord blood MDSC were characterized as positive for granulocytic markers CD66b or CD15, positive for CD33, CD11b and IL-4Ra and negative for CD14 and HLA-DR, thus classified as GRMDSC, while MO-MDSC were not elevated.

Several clinical characteristics have been evaluated as influencing factor for cord blood GR-MDSC accumulation. Most interesting, cord blood GR-MDSC levels seem to be independent of gestational age (50), resembling GR-MDSC rates in maternal blood. However, it has been reported, that very low birth weight infants, i.e. infants with a birth weight below $1500 \mathrm{~g}$ and small for gestational age (SGA) infants, i.e. weight below the $10^{\text {th }}$ percentile for the gestational age may have decreased cord blood GR-MDSC levels $(50,51)$. No influence has been described for biological sex and exposition to prenatal medication of the mother such as magnesium or corticosteroids, influencing factors that have been described for other regulatory immune effector cells such as regulatory $\mathrm{T}$ cells (Treg) in preterm infants (52).

While until now, no data on MDSC counts are available directly from the fetal blood, cord blood MDSC are regarded as of fetal origin. This is supported by the fact, that GR-MDSC have been shown to be elevated in cord blood not only from term, but also from preterm infants as early as 23 weeks of gestation (50).

Elevation of MDSC has also been described in neonatal mice in spleens and bone marrow (51), with substantial expansion of GR-MDSC up to $40 \%$ while MO-MDSC were only marginally elevated.

\section{MDSC After Birth}

After birth, different studies showed elevated GR-MDSC levels persist for at least 4 to 6 weeks, accounting a fraction of $2 \%$ to $4 \%$ of PBMC with a negative correlation of MDSC-counts and postnatal age. MDSC then further decreases to adult levels during the second month of life $(49,50)$. Paralleled to the kinetics in humans, in neonatal mice, GR-MDSC levels stay elevated during the first 3 weeks of life, then dropping to adult levels (51).

The parallelism in the kinetic of postnatal persistence of neonatal MDSC in human and mice is somehow astonishing since adaptation and maturation of various immunological functions is generally thought to follow different time scales in men and mice 
and are different in each immunological compartment such as innate cytokine response, innate myeloid cell populations and adaptive immune functions (53). For neonatal MDSC persistence external factors regulating MDSC accumulation under other circumstances such as tumor environment may be relevant. Intriguingly, reports on $\mathrm{CD}^{+} 1^{+}$erythroid cells, another immature cell population with immunosuppressive activity, which are highly elevated in cord blood and peripheral blood during the first weeks of life and thus extraordinarily mimicking the course of MDSC accumulation (54) hint toward a role of these cells for microbiome establishment after birth. Taken together, neonatal MDSC might not only be interpreted as remnant of materno-fetal tolerance and as a sign of distinct immune adaptation, but might be a crucial regulator of inflammation during the neonatal period.

\section{MDSC in Neonatal Pathologies}

Data from our group showed, that in neonates suffering from bacterial infection GR-MDSC may dramatically expand, even from the elevated level seen in healthy neonates (50), and that GR-MDSC levels correlated with inflammatory markers such as C-reactive protein (CRP), demonstrating the influence of an inflammatory environment of MDSC at least on their expansion, as seen in adult patients [reviewed in: (55)].

Interesting but somehow unclear data descend from an inflammatory disease model of neonatal mice using a gavage/ hypoxia approach to induce necrotizing enterocolitis (NEC), an often-devastating inflammatory bowel-disease of preterm infants. Selective depletion of MDSC with an agonistic antibody to TumorNecrosis-Factor-Related Apoptosis-Inducing Ligand receptor (TRAIL-R) depleted MDSC from the lamina propria and led to shortened survival and pronounced intestinal inflammation and bacterial load (51) during murine NEC. In contrast, neonatal splenic myeloid cells containing MDSC adoptively transferred intraperitoneally greatly decreased these symptoms compared to adult mouse spleen cells or mock transfer (51). These data may underline the potential role of neonatal MDSC as regulator of inflammation during the neonatal period.

\section{MDSC Functions in Neonates}

Functional characteristics of cord blood MDSC and neonatal MDSC have been described in some details and have added several new aspects in the knowledge of MDSC functionality in general (Table 2). Besides the ability to inhibit $\mathrm{T}$ cell proliferation as prerequisite and characteristic function of MDSC (48), we could demonstrate that cord blood GR-MDSC preferentially inhibited Th1 cells, contrarily polarized towards Th2 reactions (56) and induced Tregs $(51,56)$. Intriguingly these processes seemed to be mediated by different effector mechanisms. Th1 inhibition required direct cell-contact and was independent of other cell types, while induction of Th2 cells was mainly mediated through soluble factors, i.e. ArgI and ROS. Induction of Tregs was partially mediated through iNOS expression (56).

Cord blood GR-MDSC may have effects on other types of immune effector cells such as monocytes and NK-cells. In cocultures with cord blood GR-MDSC monocytes downregulated HLA class I and class II expression and upregulated co-inhibitory molecules such as programmed death ligand 1 (PD-L1) and PD-
TABLE 2 | Origin, effects, and mechanisms of MDSC in neonates.

\begin{tabular}{llll}
\hline MDSC origin & \multicolumn{1}{c}{ Effect } & \multicolumn{1}{c}{$\begin{array}{c}\text { Main mechanism } \\
\text { of MDSC }\end{array}$} & Ref. \\
\hline $\begin{array}{llll}\text { GR-MDSC from } \\
\text { cord blood }\end{array}$ & Inhibition of T-cell proliferation & - & $(44$, \\
& & & $45)$ \\
& Inhibition of Th1 responses & Cell contact & $(51)$ \\
& Induction of Th2 responses & Argl, ROS & $(51)$ \\
& Induction of Tregs & iNOS & $(47$, \\
& & & $51)$ \\
& Downregulation of HLA- & - & $(52)$ \\
& molecules on monocytes & & \\
& Upregulation of co-stimulatory & - & $(52)$ \\
& molecules PD-L1, PD-L2 & & $(52)$ \\
& Decrease of TNF-alpha, IL-1beta & - & $(52)$ \\
& Induction of IL-8 & - & \\
& Inhibition of NK-cell cytotoxicity & - &
\end{tabular}

Source of MDSC, effect on immune effector cell and the main MDSC mechanisms are listed. Argl, arginase I; ROS, reactive oxygen species; iNOS, inducible NO-synthetase.

L2, showed reduced stimulatory capacity of antigen-dependent and antigen-independent $\mathrm{T}$-cell proliferation and altered cytokine expression upon bacterial stimulation with decreased TNF- $\alpha$ and IL- $1 \beta$ but enhanced IL- 8 production (57). Taken together, monocytes seem to be biased towards an immature phenotype typically seen in cord blood monocytes (58). Furthermore, NK cell cytotoxicity was inhibited by cord blood GR-MDSC (48). On the whole cord blood GR-MDSC may orchestrate several other aspects of immune reactions in a way which is thought to be characteristic for the neonatal period.

Concerning other mechanisms for immune suppression/ modulation mediated by cord blood GR-MDSC, it could have been shown that cord blood GR-MDSC produce higher amounts of prostaglandin E2 (PGE2),express increased levels of S100A9 and lactoferrin and exert pronounced antibacterial activity compared to adult neutrophils (51).

While the functionality of neonatal MDSC has not been tested in detail in humans, mainly because of the unavailability of larger volumes of peripheral blood of neonates, function of neonatal murine GR-MDSC and MO-MDSC have been studied in-vitro and in-vivo (51). In-vitro, neonatal GR-MDSC and MO-MDSC exhibited potent suppression of antigen-specific proliferation of OT-1 CD8+ T-cells with stimulated with SIINFEKL antigen as well as antigen nonspecific CD8 T-cell proliferation stimulated with CD3/CD28. In-vivo, neonatal GR-MDSC were able to decrease lung inflammation in an ovalbumin-sensitization model (51). A summary of MDSC-mediated immune modulation in neonates is depicted in Figure 2.

\section{MDSC IN BREAST MILK}

Besides the accumulation of MDSC in the maternal organism during pregnancy as well as in the fetal and neonatal organism, recently our group could show that also breast milk contains large numbers of GR-MDSC (59). Numbers of GR-MDSC in breast milk from preterm infants correlated with gestational 


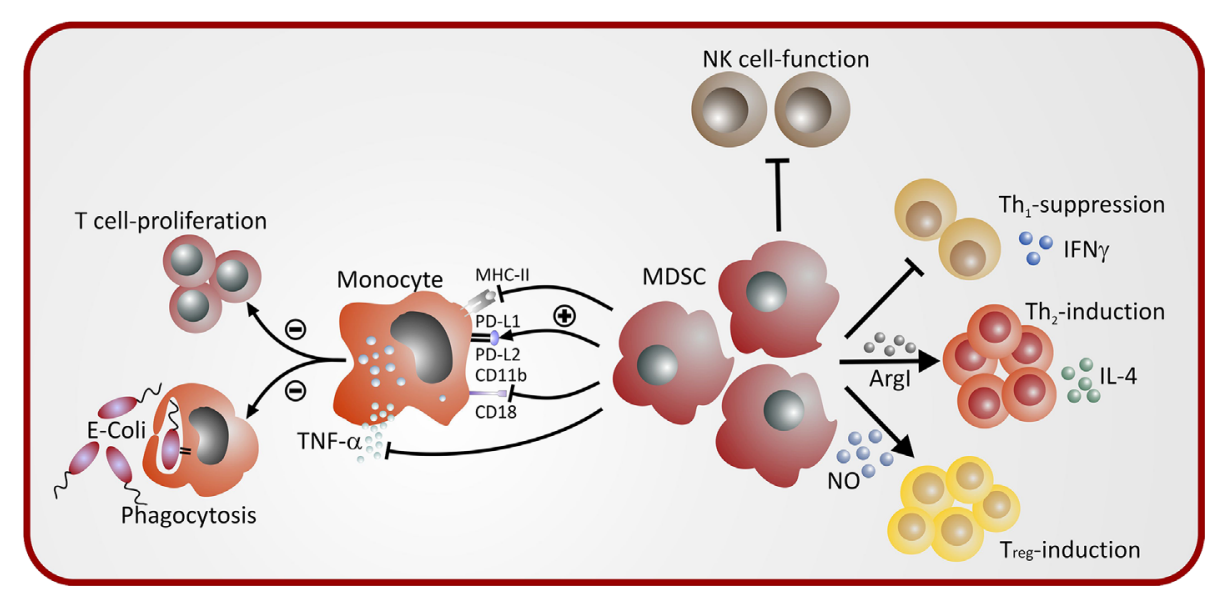

FIGURE 2 | Mechanisms of MDSC-mediated immune modulation in neonates.

age (unpublished data). In comparison to GR-MDSC from the periphery, breast milk GR-MDSC were functionally activated with increased T-cell inhibitory capacity, increased expression of CXCR4, PD-L1, PD-L2, and iNOS. Furthermore, we could show that breast milk GR-MDSC suppressed the expression of TLR-4 on monocytes. As putative mechanisms for MDSCaccumulation in breast milk, we investigated if prolactin or oxytocin could induce MDSC from PBMC, but could not show any effect (59). Beyond that, He et al. showed that breastfed infants had increased GR-MDSC levels in comparison to infants fed with formula (51). Further in-vivo studies are needed to investigate the functional role of GR-MDSC in breast milk.

\section{CONCLUSION}

Besides their well described role during pathological processes such as cancer and inflammation, where an expansion of MDSC is detrimental for the host, during the last years MDSC have been discovered also as potent regulators of critical immunological processes during pregnancy and the neonatal period such as maintenance of materno-fetal tolerance and control of inflammation in neonates. Better understanding of the differences between protective and destructive MDSCpopulations and the mechanisms leading to their expansion is

\section{REFERENCES}

1. Wegmann TG, Lin H, Guilbert L, Mosmann TR. Bidirectional cytokine interactions in the maternal-fetal relationship: is successful pregnancy a TH2 phenomenon? Immunol Today (1993) 14(7):353-6. doi: 10.1016/0167-5699(93)90235-D

2. Deshmukh H, Way SS. Immunological Basis for Recurrent Fetal Loss and Pregnancy Complications. Annu Rev Pathol (2019) 14:185-210. doi: 10.1146/ annurev-pathmechdis-012418-012743

3. Gabrilovich DI. Myeloid-Derived Suppressor Cells. Cancer Immunol Res (2017) 5(1):3-8. doi: 10.1158/2326-6066.CIR-16-0297 needed to specifically influence their functions. Targeting MDSC-functions may help to positively impact immunological pregnancy complications as well as inflammatory diseases of the newborn making them an interesting target for cellular based therapies in this field.

\section{AUTHOR CONTRIBUTIONS}

NK-G and CG both contributed to the scope of the review, literature search, and writing the manuscript. All authors contributed to the article and approved the submitted version.

\section{ACKNOWLEDGMENTS}

This research was funded by the Ministerium für Wissenschaft, Forschung und Kunst Baden-Württemberg and the European Social Fund, the German Research Foundation (DFG), grants No. KO 5272/3-1 and GI 1094/4-2 the Federal Ministry of Education and Research (BMBF), grant 01GL1745F and the German Center for Infection Research (DZIF), grant TI 07.002. We acknowledge support by Open Access Publishing Fund of University of Tübingen. We thank Peter-Michael Weber for creating the graphical illustrations.

4. Condamine T, Dominguez GA, Youn JI, Kossenkov AV, Mony S, AliceaTorres K, et al. Lectin-type oxidized LDL receptor-1 distinguishes population of human polymorphonuclear myeloid-derived suppressor cells in cancer patients. Sci Immunol (2016) 1(2). doi: 10.1126/sciimmunol.aaf8943

5. Dumitru CA, Moses K, Trellakis S, Lang S, Brandau S. Neutrophils and granulocytic myeloid-derived suppressor cells: immunophenotyping, cell biology and clinical relevance in human oncology. Cancer Immunol Immunother (2012) 61(8):1155-67. doi: 10.1007/s00262-012-1294-5

6. Bronte V, Brandau S, Chen SH, Colombo MP, Frey AB, Greten TF, et al. Recommendations for myeloid-derived suppressor cell nomenclature and 
characterization standards. Nat Commun (2016) 7:12150. doi: 10.1038/ ncomms 12150

7. Shipp C, Speigl L, Janssen N, Martens A, Pawelec G. A clinical and biological perspective of human myeloid-derived suppressor cells in cancer. Cell Mol Life Sci CMLS (2016) 73(21):4043-61. doi: 10.1007/s00018-016-2278-y

8. Mauti LA, Le Bitoux MA, Baumer K, Stehle JC, Golshayan D, Provero P, et al. Myeloid-derived suppressor cells are implicated in regulating permissiveness for tumor metastasis during mouse gestation. J Clin Invest (2011) 121 (7):2794-807. doi: 10.1172/JCI41936

9. Poschke I, Mao Y, Adamson L, Salazar-Onfray F, Masucci G, Kiessling R. Myeloid-derived suppressor cells impair the quality of dendritic cell vaccines. Cancer Immunol Immunother (2012) 61(6):827-38. doi: 10.1007/s00262-0111143-y

10. Sinha P, Clements VK, Bunt SK, Albelda SM, Ostrand-Rosenberg S. Crosstalk between myeloid-derived suppressor cells and macrophages subverts tumor immunity toward a type 2 response. J Immunol (2007) 179(2):977-83. doi: 10.4049/jimmunol.179.2.977

11. Hoechst B, Ormandy LA, Ballmaier M, Lehner F, Kruger C, Manns MP, et al. A new population of myeloid-derived suppressor cells in hepatocellular carcinoma patients induces CD $4(+) \mathrm{CD} 25(+)$ Foxp3(+) T cells. Gastroenterology (2008) 135(1):234-43. doi: 10.1053/j.gastro.2008.03.020

12. Condamine T, Mastio J, Gabrilovich DI. Transcriptional regulation of myeloid-derived suppressor cells. J Leukoc Biol (2015) 98(6):913-22. doi: 10.1189/jlb.4RI0515-204R

13. Youn JI, Nagaraj S, Collazo M, Gabrilovich DI. Subsets of myeloid-derived suppressor cells in tumor-bearing mice. J Immunol (2008) 181(8):5791-802. doi: 10.4049/jimmunol.181.8.5791

14. Pawelec G, Verschoor CP, Ostrand-Rosenberg S. Myeloid-Derived Suppressor Cells: Not Only in Tumor Immunity. Front Immunol (2019) 10:1099. doi: 10.3389/fimmu.2019.01099

15. Kostlin N, Kugel H, Spring B, Leiber A, Marme A, Henes M, et al. Granulocytic myeloid derived suppressor cells expand in human pregnancy and modulate T-cell responses. Eur J Immunol (2014) 44(9):2582-91. doi: $10.1002 /$ eji.201344200

16. Nair RR, Sinha P, Khanna A, Singh K. Reduced Myeloid-derived Suppressor Cells in the Blood and Endometrium is Associated with Early Miscarriage. Am J Reprod Immunol (2014) 73(6):479-86. doi: 10.1111/aji.12351

17. Kostlin N, Hofstadter K, Ostermeir AL, Spring B, Leiber A, Haen S, et al. Granulocytic Myeloid-Derived Suppressor Cells Accumulate in Human Placenta and Polarize toward a Th2 Phenotype. J Immunol (2016) 196 (3):1132-45. doi: 10.4049/jimmunol.1500340

18. Bartmann C, Junker M, Segerer SE, Hausler SF, Krockenberger M, Kammerer U. CD33(+) /HLA-DR(neg) and CD33(+) /HLA-DR(+/-) Cells: Rare Populations in the Human Decidua with Characteristics of MDSC. Am J Reprod Immunol (2016) 75(5):539-56. doi: 10.1111/aji.12492

19. Pan T, Zhong L, Wu S, Cao Y, Yang Q, Cai Z, et al. 17beta-estradiol enhances the expansion and activation of myeloid-derived suppressor cells via STAT3 signaling in human pregnancy. Clin Exp Immunol (2016) 100(3):499-511. doi: $10.1111 /$ cei. 12790

20. Zhang Y, Qu D, Sun J, Zhao L, Wang Q, Shao Q, et al. Human trophoblast cells induced MDSCs from peripheral blood CD14(+) myelomonocytic cells via elevated levels of CCL2. Cell Mol Immunol (2016) 13(5):615-27. doi: $10.1038 / \mathrm{cmi} .2015 .41$

21. Ostrand-Rosenberg S, Sinha P, Figley C, Long R, Park D, Carter D, et al. Frontline Science: Myeloid-derived suppressor cells (MDSCs) facilitate maternal-fetal tolerance in mice. J Leukoc Biol (2017) 101(5):1091-101. doi: 10.1189/jlb.1HI1016-306RR

22. Kostlin-Gille N, Dietz S, Schwarz J, Spring B, Pauluschke-Frohlich J, Poets CF, et al. HIF-1alpha-Deficiency in Myeloid Cells Leads to a Disturbed Accumulation of Myeloid Derived Suppressor Cells (MDSC) During Pregnancy and to an Increased Abortion Rate in Mice. Front Immunol (2019) 10:161. doi: 10.3389/fimmu.2019.00161

23. Zhao H, Kalish F, Schulz S, Yang Y, Wong RJ, Stevenson DK. Unique Roles of Infiltrating Myeloid Cells in the Murine Uterus during Early to Midpregnancy. J Immunol (2015) 194(8):3713-22. doi: 10.4049/ jimmunol.1401930

24. Kang X, Zhang X, Liu Z, Xu H, Wang T, He L, et al. Granulocytic myeloidderived suppressor cells maintain feto-maternal tolerance by inducing Foxp3 expression in CD4+CD25-T cells by activation of the TGF-beta/beta-catenin pathway. Mol Hum Reprod (2016) 22(7):499-511. doi: 10.1093/molehr/ gaw026

25. Pan T, Liu Y, Zhong LM, Shi MH, Duan XB, Wu K, et al. Myeloid-derived suppressor cells are essential for maintaining feto-maternal immunotolerance via STAT3 signaling in mice. J Leukoc Biol (2016) 100(3):499-511. doi: 10.1189/jlb.1A1015-481RR

26. Verma P, Verma R, Nair RR, Budhwar S, Khanna A, Agrawal NR, et al. Altered crosstalk of estradiol and progesterone with Myeloid-derived suppressor cells and Th1/Th2 cytokines in early miscarriage is associated with early breakdown of maternal-fetal tolerance. Am J Reprod Immunol (2019) 81(2):e13081. doi: 10.1111/aji.13081

27. Kang X, Zhang X, Liu Z, Xu H, Wang T, He L, et al. CXCR2-Mediated Granulocytic Myeloid-Derived Suppressor Cells' Functional Characterization and Their Role in Maternal Fetal Interface. DNA Cell Biol (2016) 35(7):35865. doi: 10.1089/dna.2015.2962

28. Zhu M, Huang X, Yi S, Sun H, Zhou J. High granulocytic myeloid-derived suppressor cell levels in the peripheral blood predict a better IVF treatment outcome. J Matern Fetal Neonatal Med (2019) 32(7):1092-7. doi: 10.1080/ 14767058.2017.1400002

29. Hu C, Zhen Y, Pang B, Lin X, Yi H. Myeloid-Derived Suppressor Cells Are Regulated by Estradiol and Are a Predictive Marker for IVF Outcome. Front Endocrinol (Lausanne) (2019) 10:521. doi: 10.3389/fendo.2019.00521

30. Wang Y, Liu Y, Shu C, Wan J, Shan Y, Zhi X, et al. Inhibition of pregnancyassociated granulocytic myeloid-derived suppressor cell expansion and arginase-1 production in preeclampsia. J Reprod Immunol (2018) 127:4854. doi: 10.1016/j.jri.2018.05.002

31. Kropf P, Baud D, Marshall SE, Munder M, Mosley A, Fuentes JM, et al. Arginase activity mediates reversible $\mathrm{T}$ cell hyporesponsiveness in human pregnancy. Eur J Immunol (2007) 37(4):935-45. doi: 10.1002/eji.200636542

32. Ren J, Zeng W, Tian F, Zhang S, Wu F, Qin X, et al. Myeloid-derived suppressor cells depletion may cause pregnancy loss via upregulating the cytotoxicity of decidual natural killer cells. Am J Reprod Immunol (2019) 81 (4):e13099. doi: 10.1111/aji.13099

33. Erlebacher A. Mechanisms of $\mathrm{T}$ cell tolerance towards the allogeneic fetus. Nat Rev Immunol (2013) 13(1):23-33. doi: 10.1038/nri3361

34. Nagaraj S, Gupta K, Pisarev V, Kinarsky L, Sherman S, Kang L, et al. Altered recognition of antigen is a mechanism of $\mathrm{CD} 8+\mathrm{T}$ cell tolerance in cancer. Nat Med (2007) 13(7):828-35. doi: 10.1038/nm1609

35. Sykes L, MacIntyre DA, Yap XJ, Teoh TG, Bennett PR. The Th1:th2 dichotomy of pregnancy and preterm labour. Mediators Inflamm (2012) 2012:967629. doi: 10.1155/2012/967629

36. Aluvihare VR, Kallikourdis M, Betz AG. Regulatory T cells mediate maternal tolerance to the fetus. Nat Immunol (2004) 5(3):266-71. doi: 10.1038/ni1037

37. Bulmer JN, Williams PJ, Lash GE. Immune Cells placental bed. Int J Dev Biol (2010) 54(2-3):281-94. doi: 10.1387/ijdb.082763jb

38. Trundley A, Moffett A. Human uterine leukocytes and pregnancy. Tissue Antigens (2004) 63(1):1-12. doi: 10.1111/j.1399-0039.2004.00170.x

39. Elkabets M, Ribeiro VS, Dinarello CA, Ostrand-Rosenberg S, Di Santo JP, Apte RN, et al. IL-1beta regulates a novel myeloid-derived suppressor cell subset that impairs NK cell development and function. Eur J Immunol (2010) 40(12):3347-57. doi: 10.1002/eji.201041037

40. Liu C, Yu S, Kappes J, Wang J, Grizzle WE, Zinn KR, et al. Expansion of spleen myeloid suppressor cells represses NK cell cytotoxicity in tumor-bearing host. Blood (2007) 109(10):4336-42. doi: 10.1182/blood-2006-09-046201

41. Arck PC, Hecher K. Fetomaternal immune cross-talk and its consequences for maternal and offspring's health. Nat Med (2013) 19(5):548-56. doi: 10.1038/ nm. 3160

42. Hu CE, Gan J, Zhang RD, Cheng YR, Huang GJ. Up-regulated myeloidderived suppressor cell contributes to hepatocellular carcinoma development by impairing dendritic cell function. Scand J Gastroenterol (2011) 46(2):15664. doi: $10.3109 / 00365521.2010 .516450$

43. Kostlin N, Ostermeir AL, Spring B, Schwarz J, Marme A, Walter CB, et al. HLA$\mathrm{G}$ promotes myeloid-derived suppressor cell accumulation and suppressive activity during human pregnancy through engagement of the receptor ILT4 Eur J Immunol (2017) 47(2):374-84. doi: 10.1002/eji.201646564

44. Wenger RH. Cellular adaptation to hypoxia: O2-sensing protein hydroxylases, hypoxia-inducible transcription factors, and O2-regulated 
gene expression. FASEB J (2002) 16(10):1151-62. doi: 10.1096/fj.010944rev

45. Zenclussen ML, Casalis PA, El-Mousleh T, Rebelo S, Langwisch S, Linzke N, et al. Haem oxygenase-1 dictates intrauterine fetal survival in mice via carbon monoxide. J Pathol (2011) 225(2):293-304. doi: 10.1002/path.2946

46. Zhao H, Kalish F, Wong RJ, Stevenson DK. Infiltration of myeloid cells in the pregnant uterus is affected by heme oxygenase-1. J Leukoc Biol (2017) 101 (1):217-26. doi: 10.1189/jlb.1A0116-020RR

47. De Wilde V, Van Rompaey N, Hill M, Lebrun JF, Lemaitre P, Lhomme F, et al. Endotoxin-induced myeloid-derived suppressor cells inhibit alloimmune responses via heme oxygenase-1. Am J Transplant (2009) 9(9):2034-47. doi: 10.1111/j.1600-6143.2009.02757.x

48. Rieber N, Gille C, Kostlin N, Schafer I, Spring B, Ost M, et al. Neutrophilic myeloid-derived suppressor cells in cord blood modulate innate and adaptive immune responses. Clin Exp Immunol (2013) 174(1):45-52. doi: 10.1111/cei.12143

49. Gervassi A, Lejarcegui N, Dross S, Jacobson A, Itaya G, Kidzeru E, et al. Myeloid derived suppressor cells are present at high frequency in neonates and suppress in vitro T cell responses. PLoS One (2014) 9(9):e107816. doi: 10.1371/journal.pone.0107816

50. Schwarz J, Scheckenbach V, Kugel H, Spring B, Pagel J, Hartel C, et al. Granulocytic myeloid-derived suppressor cells (GR-MDSC) accumulate in cord blood of preterm infants and remain elevated during the neonatal period. Clin Exp Immunol (2018) 191(3):328-37. doi: 10.1111/cei.13059

51. He YM, Li X, Perego M, Nefedova Y, Kossenkov AV, Jensen EA, et al. Transitory presence of myeloid-derived suppressor cells in neonates is critical for control of inflammation. Nat Med (2018) 24(2):224-31. doi: 10.1038/nm.4467

52. Luciano AA, Arbona-Ramirez IM, Ruiz R, Llorens-Bonilla BJ, MartinezLopez DG, Funderburg N, et al. Alterations in regulatory $\mathrm{T}$ cell subpopulations seen in preterm infants. PLoS One (2014) 9(5):e95867. doi: 10.1371/journal.pone.0095867

53. Dowling DJ, Levy O. Ontogeny of early life immunity. Trends Immunol (2014) 35(7):299-310. doi: 10.1016/j.it.2014.04.007

54. Elahi S, Ertelt JM, Kinder JM, Jiang TT, Zhang X, Xin L, et al. Immunosuppressive CD71+ erythroid cells compromise neonatal host defence against infection. Nature (2013) 504(7478):158-62. doi: 10.1038/ nature 12675

55. Schrijver IT, Theroude C, Roger T. Myeloid-Derived Suppressor Cells in Sepsis. Front Immunol (2019) 10:327. doi: 10.3389/fimmu.2019.00327

56. Kostlin N, Vogelmann M, Spring B, Schwarz J, Feucht J, Hartel C, et al. Granulocytic myeloid-derived suppressor cells from human cord blood modulate T-helper cell response towards an anti-inflammatory phenotype. Immunology (2017) 152(1):89-101. doi: 10.1111/imm.12751

57. Dietz S, Schwarz J, Vogelmann M, Spring B, Molnar K, Orlikowsky TW, et al. Cord blood granulocytic myeloid-derived suppressor cells impair monocyte T cell stimulatory capacity and response to bacterial stimulation. Pediatr Res (2019) 86(5):608-15. doi: 10.1038/s41390-019-0504-7

58. Orlikowsky TW, Spring B, Dannecker GE, Niethammer D, Poets CF, Hoffmann MK. Expression and regulation of B7 family molecules on macrophages (MPhi) in preterm and term neonatal cord blood and peripheral blood of adults. Cytometry B Clin Cytom (2003) 53(1):40-7. doi: $10.1002 /$ cyto.b.10033

59. Kostlin N, Schoetensack C, Schwarz J, Spring B, Marme A, Goelz R, et al. Granulocytic Myeloid-Derived Suppressor Cells (GR-MDSC) in Breast Milk (BM); GR-MDSC Accumulate in Human BM and Modulate T-Cell and Monocyte Function. Front Immunol (2018) 9:1098. doi: 10.3389/ fimmu.2018.01098

Conflict of Interest: The authors declare that the research was conducted in the absence of any commercial or financial relationships that could be construed as a potential conflict of interest.

Copyright $\odot 2020$ Köstlin-Gille and Gille. This is an open-access article distributed under the terms of the Creative Commons Attribution License (CC BY). The use, distribution or reproduction in other forums is permitted, provided the original author(s) and the copyright owner(s) are credited and that the original publication in this journal is cited, in accordance with accepted academic practice. No use, distribution or reproduction is permitted which does not comply with these terms. 